\title{
CORRECTIONS TO THE STERMAN-WEINBERG JET FORMULA
}

\author{
Bruce G. WEEKS \\ Randall Laboratory of Physics, University of Michigan, Ann Arbor, MI 48109, USA
}

Received 24 November 1978

The fraction of hadronic final states from $\mathrm{e}^{+} \mathrm{e}^{-}$annihilation consisting of two jets is calculated exactly to lowest order in QCD perturbation theory. This improves the Sterman-Weinberg expression increasing its range of applicability and may allow for detailed quantitative tests of QCD at feasible experimental energies.

The most straightforward way to obtain experimental predictions from a lagrangian field theory is by means of Feynman diagrammatic perturbation theory. In the case of quantum chromodynamics, such predictions are often invalidated by a large coupling constant, by mass singularities, and by the nonperturbative nature of confinement. Lately, quantities free of the first two difficulties (and for which, we hope, the third difficulty may be ignored) have been investigated as a means of obtaining detailed numerical predictions from QCD [1-3].

One such quantity is the fraction $f$ of final states from $\mathrm{e}^{+} \mathrm{e}^{-}$annihilation which consists of "two jet" events. A two jet event, as defined by Sterman and Weinberg [1], has all but a fraction $\epsilon$ of the total energy $E$ going into some pair of cones each of half-angle $\delta$. Sterman and Weinberg derived

$1-f=\left(\alpha_{\mathrm{s}} / \pi\right) \cdot \frac{4}{3} \cdot Q(\delta, \epsilon)+\mathrm{O}\left(\alpha_{\mathrm{s}}^{2}\right)$

with

$Q(\delta, \epsilon)=4 \log \delta^{-1}\left[\log (2 \epsilon)^{-1}-\frac{3}{4}\right]+\pi^{2} / 3-\frac{7}{4}$,

where terms vanishing as $\delta, \epsilon \rightarrow 0$ have been dropped. $\alpha_{\mathrm{s}} \equiv g^{2} / 4 \pi$, with $g$ being the value of the strong coupling appropriate to this process. More will be said about the value of $\alpha_{s}$ later.

The predictions represented by eqs. (1) and (2) may provide detailed tests of QCD as the theory of

\footnotetext{
Work supported by the Department of Energy and by a University of Michigan Rackham Predoctoral Fellowship.
}

strong interactions. Unfortunately, as noted elsewhere $[3,4]$, the formulas above are of limited usefulness. At any energies which will be experimentally accessible in the reasonably near future, $1-f$ becomes sizeable for small $\delta$ and $\epsilon$, signalling the inapplicability of perturbation theory. For $\delta$ and $\epsilon$ large, the terms which vanished as $\delta, \epsilon \rightarrow 0$ and were neglected could be significant. In this note, we eliminate the latter difficulty by computing $1-f$ exactly (to order $\alpha_{s}$ ). (This extends the result of Stevenson $[4]{ }^{\neq 1}$.) We then consider possible applications of the new result.

As noted elsewhere $[3,4] 1-f$ is proportional to the integral of the $e^{+} e^{-} \rightarrow q \bar{q} g$ matrix element squared over that part of the final state phase space not consisting of 2 -jet events. Let $x, y, z$ be the $\mathrm{q}, \overline{\mathrm{q}}, \mathrm{g}$ energies measured in units of $\frac{1}{2} E$. After integrating over various orientations of the final state, $1-f$ is proportional to $[4,5]$.

$\int_{\mathrm{R}} \mathrm{d} x \mathrm{~d} y\left(x^{2}+y^{2}\right) /(1-x)(1-y)$.

The region $\mathrm{R}$ is shown in fig. 1 along with a subregion $\mathrm{A}$ which we use below. Since all three final particles are taken to be massless, phase space is invariant under particle interchange, and we may symmetrize the integrand in $x, y$, and $z$ without changing the result. Having done so, we may restrict the phase space

\footnotetext{
₹1 Actually, expanding our eq. (3) leads to a slight disagreement with this author.
} 
region by requiring $x<y<z$. This yields

$$
\begin{aligned}
1-\lambda & \propto \int_{\mathrm{A}} \mathrm{d} x \mathrm{~d} y\left[\frac{x^{2}+y^{2}}{(1-x)(1-y)}\right. \\
& \left.+\frac{x^{2}+z^{2}}{(1-x)(1-z)}+\frac{y^{2}+z^{2}}{(1-y)(1-z)}\right],
\end{aligned}
$$

where $x+y+z=2$. Furthermore, straightforward kinematics yields

$$
\begin{aligned}
& \int_{A} \mathrm{~d} x \mathrm{~d} y=\int_{2 \epsilon}^{1 / 2+b} \mathrm{~d} x \int_{(1-x) /(1-a x)}^{1-x / 2} \mathrm{~d} y \\
& +\int_{1 / 2+b}^{2 / 3} \mathrm{~d} x \int_{x}^{1-x / 2} \mathrm{~d} y,
\end{aligned}
$$

where $a \equiv \frac{1}{2}(1-\cos 2 \delta)=\sin ^{2} \delta$ and $b \equiv(1-\sqrt{1-a}) / a$ $-\frac{1}{2}=\frac{1}{2} \tan ^{2}\left(\frac{1}{2} \delta\right)$.

The integrals can now be performed. The result is eq. (1), with eq. (2) replaced with

$$
\begin{aligned}
& Q(\delta, \epsilon)=2 \log [(1-a) / a] \\
& \quad \times\left[\log (2 \epsilon)^{-1}-\frac{3}{4}+\log (1-2 \epsilon)+3 \epsilon\right. \\
& \left.\quad+\log (1+2 b) /(1-2 b)-\frac{3}{2} b\right]+\pi^{2} / 3-\frac{7}{4} \\
& \quad-2 \mathcal{L}_{2}(2 \epsilon)-\log ^{2}(1-2 \epsilon) \\
& \quad+\left(\frac{3}{2}-6 \epsilon\right) \log (1-2 \epsilon)+3 \epsilon+3 \epsilon^{2} \\
& \quad+\left.4 \mathcal{L}_{2}\right|_{1 / 2} ^{1 / 2+b}+2 \mathcal{L}_{2}(-2 b)-2 \mathcal{L}_{2}(2 b) \\
& \quad+\frac{3}{2} b-\frac{3}{2} b^{2}-\left.2 \log ^{2}\right|_{1 / 2-b} ^{1 / 2} \\
& \quad+[2 \log (1+2 b) /(1-2 b)-3 b] \log 2 b \\
& \quad+\left.6 x \log x\right|_{1 / 2-b} ^{1 / 2}+\left.\left[3 a x^{2}+\left(2 a^{2}-a\right) x^{3} r(a x)\right]\right|_{2 \epsilon} ^{1 / 2+b}
\end{aligned}
$$

where $\left.f(x)\right|_{r} ^{s}=\left.f\right|_{r} ^{s} \equiv f(s)-f(r) . \mathcal{L}_{2}$ is the dilogarithm, and $-\log (1-x)=x+\frac{1}{2} x^{2}+\frac{1}{3} x^{3} r(x)$ defines $r(x)$.

The solid lines in fig. 2 display $Q$ as a function of $\delta$ for several values of $\epsilon$. The dashed lines are the original Sterman-Weinberg result, which is seen over much of the plot to be a better approximation than might have been expected a priori.

Before applying eqs. (1) and (3), we need to consider $\alpha_{\mathrm{s}} / \pi$. The only candidate which presents itself is the running coupling constant evaluated at energy $E$, which we will denote as $\alpha_{\mathrm{s}}(E)$. Setting $\alpha_{\mathrm{s}}=\alpha_{\mathrm{s}}(E)$, we have at very high energies the result [6]

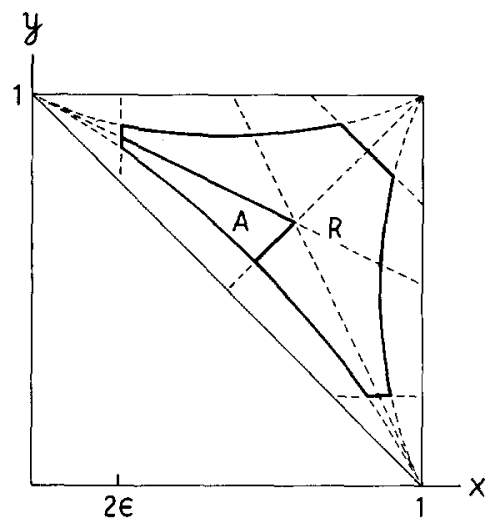

Fig. 1. Phase space regions of interest. $R$ is that region whose complement consists of 2-jet events. $\mathrm{A}$ is the subregion of $\mathrm{R}$ satisfying $x<y<z=2-x-y$.

$\frac{\alpha_{\mathrm{s}}}{\pi}=\frac{6}{(33-2 N)} \frac{1}{\log (E / \Lambda)}$,

where $N$ is the number of quark flavors and $\Lambda$ is some not too small scale with $\Lambda \ll E$. Although it is not immediately clear how to extrapolate eq. (4) to experi-

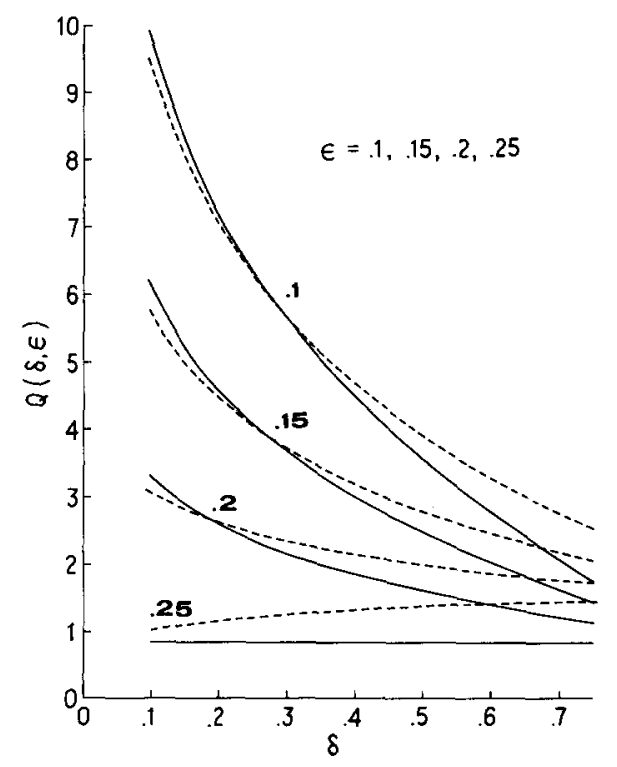

Fig. 2. $Q(\delta, \epsilon)$, which contains all the $\delta$ and $\epsilon$ dependence of $1-f$, as a function of $\delta$ for several values of $\epsilon$. The solid lines are the exact result; the dashed lines are the original StermanWeinberg result. 
mentally accessible energies, there is experimental evidence [7] that eq. (4) works quite well in describing scaling violations in deep inelastic scattering with $N$ $=4$ and $\Lambda=0.5 \mathrm{GeV}$. The corresponding prediction for $\mathrm{e}^{+} \mathrm{e}^{-}$annihilation is in reasonable agreement with the observed value of the total cross section above charm threshold [8]. We therefore optimistically take eq. (4) to hold in general, with $N$ and $\Lambda$ being energy independent between flavor thresholds.

We can now apply eqs. (1) and (3) to experiment. These equations contain a lot of information, most of which could not be obtained without the specific application of QCD. Thus, they check QCD directly. For example, with fixed $\delta$ and $\epsilon, 1-\lambda$ can be measured as a function of energy, although the preceding paragraphs indicate that one should be cautious with these predictions. On the other hand, for fixed energy ( $E=30$ $\mathrm{GeV}$ would be nice), eqs. (1) and (3) should accurately describe the variation of $1-\lambda$ with $\delta$ and $\epsilon$. Thus, it is hoped that the results shown in fig. 2 can be tested experimentally, yielding a practical quantitative test of QCD.

My thanks to Martin B. Einhorn for several indispensable discussions.

\section{References}

[1] G. Sterman and S. Weinberg, Phys. Rev. Lett. 39 (1977) 1436.

[2] C.L. Basham, L.S. Brown, S.D. Ellis and S.T. Lore, Univ. of Washington RLO-1388-746 (1977); So-Young Pi, R.L. Jaffe and F.E. Low, MIT, CTP 715 (June 1978);

K. Shizaya and S.-H.H. Tye, FERMILAB-PUB-78/54-THY (June 1978).

[3] M.B. Einhorn and B.G. Weeks, SLAC-PUB-2164 (July 1978).

[4] P.M. Stevenson, Imperial College, ICTP/77-78/25 (July 1978).

[5] T.A. DeGrand, Y.J. Ng and S.-H.H. Tye, Phys. Rev. D16 (1977) 3251

J. Ellis, M.K. Gaillard and G.G. Ross, Nucl. Phys. B111 (1976) 253.

[6] D. Gross and F. Wilczek, Phys. Rev. Lett. 26 (1973) 1343; H.D. Politzer, Phys. Rev. Lett. 26 (1973) 1346.

[7] See, e.g., the review by G.C. Fox, invited talk presented at the Intern. Conf. on Neutrino physics and neutrino astrophysics (Lafayette, April 28 to May 2, 1978), unpublished.

[8] See, e.g., the review by G. Feldman, invited talk presented at XIX Intern. Conf. on High energy physics (Tokyo, Aug. 23-30, 1978), unpublished. 